\title{
Three-dimensional hydraulic conductivity upscaling in groundwater modeling
}

\author{
Haiyan Zhou*, Liangping Li, J. Jaime Gómez-Hernández \\ Group of Hydrogeology, Department of hydraulics and environmental engineering, Universidad Politécnica de \\ Valencia, Camino de Vera, $s / n$, 46022, Spain
}

\begin{abstract}
The main point of this paper is to propose a non-local three-dimensional hydraulic conductivity full tensor upscaling algorithm and code. The algorithm is capable of transforming very refined cell conductivity models into coarse block conductivity models for quick and accurate solution of the groundwater flow equation. Flow rate and hydraulic head gradient are the variables used to relate the outputs from the fine scale model to the outputs from the coarse scale model. The flows and gradients computed at the coarse scale blocks should match the average values of the corresponding quantities observed at the fine scale. The algorithm is geared towards its use in conjunction with finite-difference codes for the solution of the groundwater flow equation capable of handling full tensor hydraulic conductivities. The finite-difference formulation of the groundwater flow equation requires specifying the hydraulic conductivity at the block interface in order to compute the discharge crossing the interface. Most finite-difference codes take as input conductivities at the blocks and then perform some type of averaging to come up with the interblock conductivity. Typically the harmonic mean of the conductivities of neighboring blocks is used. We propose computing directly the interblock conductivity during the upscaling process thus avoiding the need to average already averaged (upscaled) values of adjacent blocks. This approach also circumvents the problem associated with averaging conductivity tensors when their principal directions are not aligned with the Cartesian axes. The proposed algorithm is successfully demonstrated in four synthetic examples with spatially isotropic, anisotropic and bimodal conductivities fields at the fine scale. The computer code is provided with explanation of the input parameters and output results.
\end{abstract}

Key words: Upscaling; Full tensor; Block conductivity; Interface; Heterogeneity; Groundwater simulation.

\section{Introduction}

Numerical simulation of groundwater flow and solute transport is nowadays widely employed in predicting available groundwater resources and the fate of pollutant plumes. Hydraulic conductivity is, without any doubt, the dominant parameter in the numerical simulations. However, hydraulic conductivities are measured in the field on a support scale which is much smaller than the discretization scale of the numerical model. Dagan (1986) discusses three support scales: the laboratory, the local, and the regional scale. Transforming hydraulic conductivities from a fine scale onto a coarser scale is termed upscaling and has been the subject of research for many years (not only with regard to hydraulic conductivity but also with regard to other parameters and in other disciplines). The need for upscaling stems from the fact that it is possible to characterize the spatial variability of hydraulic conductivity at very small scales, yet, it is prohibitive to perform numerical simulations at such scale, particularly in the context of sensitivity studies, inverse modeling or Monte-Carlo analyses, which require multiple runs of the computer codes. The reader is referred to many

\footnotetext{
${ }^{*}$ Corresponding author

Email addresses: haizh@upvnet.upv.es (Haiyan Zhou), liali@upvnet.upv.es (Liangping Li), jaime@dihma.upv.es (J. Jaime Gómez-Hernández)
} 
reviews about upscaling which are available (e.g., Wen and Gómez-Hernández, 1996; Renard and Marsily, 1997; Farmer, 2002; Sanchez-Vila et al., 2006).

Several terms are used in the literature in relation to conductivity upscaling: effective hydraulic conductivity, equivalent conductivity, interpreted conductivity, upscaled conductivity, block conductivity, homogenized conductivity, ..., of which effective conductivity and equivalent conductivity are the most common. Effective conductivity is defined, from a stochastic point of view, through the equation, $E\{q\}=-K_{e f f} E\{\nabla h\}$ (Matheron, 1967), where $q$ is specific discharge, $E\{\cdot\}$ indicates expected value computed through an ensemble of realizations at a given location, and $\nabla h$ is the hydraulic head gradient. On the other hand, equivalent conductivity represents an average value of hydraulic conductivity within a given block in a given realization. Equivalent conductivities are computed to satisfy certain criteria, such as flow conservation, head reproduction or energy dissipation (Renard and Marsily, 1997; Vermeulen et al., 2006). Under certain conditions, effective conductivity and equivalent conductivity coincide; this happens when the random function describing hydraulic conductivity is stationary, ergodic, satisfy some symmetry properties, and the block size for which the equivalent conductivity is sought is much larger than the scale of correlation of the underlying random function (Matheron, 1967).

We are interested in proposing an operational solution to an engineering problem, which could be used in the most generic conditions. Nowadays, characterization techniques allow collecting data at very small scales and building heterogeneous model descriptions of multi-million nodes. Hydrogeologists and engineers face then the problem of having to solve the groundwater flow equation on these models. In many occasions, if a sensitivity analysis is to be performed, or some kind of inverse modeling (or history matching, as termed by petroleum engineers), such a solution has to be obtained in hundreds or thousands of realizations of these heterogeneous parameters. Being capable of performing flow simulations on so many realizations is unfeasible unless some type of reduction of the size of the problem is achieved (Nœetinger et al., 2005). For this reason we have analyzed the problem from an engineering perspective in order to provide a solution and a computer code that will alleviate the computational burden of having to solve the groundwater flow equation on many realizations of multimillion discretized cells. We adopt a rigorous approach understanding that filtering out some of the heterogeneity when going from a fine scale model up to a coarser model requires considering hydraulic conductivities as tensors (Lake, 1988). Tensors with principal directions not necessarily aligned with the Cartesian axes, nor necessarily equal in all the coarse blocks. The coarse scale conductivity tensors should result in a solution of the groundwater flow equation that will reproduce as close as possible the fluxes and piezometric heads observed in the fine scale model.

We are aware of the many analytical attempts to determine block conductivities representative of heterogeneous media, which basically start with the same premise as this work, i.e., to determine a block values that reproduce the behavior of the heterogeneous medium at the scale of the upscaled blocks. Significant works within the realm of hydrogeology are those by Dagan (1989); Rubin and Gómez-Hernández (1990); Indelman and Dagan (1993); Neuman and Orr (1993). The problem of upscaling is not exclusive to groundwater flow, and it has also been addressed in other fields, sometimes with a different terminology. For instance, the renormalization group theories from statistical physics have been successfully applied for conductivity upscaling (Gautier and Noetinger, 1997), and recently, there have been some promising works with the filtering approaches used in large eddy simulations by Attinger and co-workers (Attinger et al., 2002). All of these works provide insights on the type of upscaled conductivity descriptions that best reproduce the fine scale simulations, yet, they are limited to very specific spatial patterns for the underlying hydraulic conductivity distribution and very simple aquifer geometries.

There are some analytical solutions that have proven exact, or very accurate, in practical situations. For instance, in one-dimensional flow problems, it is well known that the harmonic mean is the exact equivalent conductivity (e.g., Freeze and Cherry, 1979), whereas in two-dimensional perfectly layered media, the equivalent conductivity is a tensor with principal directions parallel and orthogonal to the layer orientation, with the arithmetic mean being the component parallel to the layering and the harmonic mean the component in the orthogonal direction. In two-dimensional flow, for conductivities with an isotropic spatial correlation and lognormal probability distribution, and for blocks sufficiently large, the geometric mean is a good approximate for the block value (Matheron, 1967; Gómez-Hernández and Wen, 1994). There is even 
a conjecture derived by Landau and Lifshitz (1960) coming from the electrodynamics field that has been utilized in groundwater flow. This conjecture gives a general expression for the block conductivities (for sufficiently large blocks) for an isotropically correlated spatial distribution and for any space dimensionality; this expression returns the harmonic mean for 1-D problems, and the geometric mean for 2-D problems, the 3-D results have been verified in moderately heterogeneous fields by Gelhar and Axness (1983), and in large variance fields by Neuman and Orr (1993).

Our work should be framed within the work started by Rubin and Gómez-Hernández (1990) in which Darcy's law is spatially integrated within the block being upscaled, and the block conductivity is defined as the tensor that best relates the average head gradient to the average specific discharge vector (see below, Equation (1)). In this averaging process we recognize that the resulting upscaled values depend on the boundary conditions used to compute them, that is, the block conductivities are non-local and depend not only on the conductivities within the block, but also on the boundary conditions around the block.

Our approach, being numeric, is not limited by a specific spatial distribution pattern, nor the degree of variability, nor the geometry of the aquifer, nor the boundary conditions governing the groundwater flow problem. We use the best possible benchmark to verify the accuracy of our method: the solution at the fine scale that we would like to reproduce at the coarse scale.

We are interested in replacing an aquifer model of many cells with a coarser model with a few thousand blocks. Each coarse block will replace a set of fine cells. Each block will be assigned an equivalent conductivity with the aim of reproducing as best as possible the flow and piezometric head patters of the fine model by the coarse one.

The objective of this paper is to present a very accurate three-dimensional full-tensor hydraulic conductivity upscaling algorithm and code aimed to derive interblock hydraulic conductivity tensors for direct use in a finite-difference groundwater flow simulation program. Next, the algorithm is described, then four synthetic cases are presented, and the paper ends with some conclusions.

\section{Algorithm and implementation}

\subsection{Upscaling methodology}

Following Rubin and Gómez-Hernández (1990) we define block conductivity $\left(\vec{K}_{V}\right)$ as the quantity that relates the average specific discharge within a given block to the average head gradient as follows:

$$
\frac{1}{V} \int_{V} \vec{q} d V=-\vec{K}_{V}\left(\frac{1}{V} \int_{V} \overrightarrow{\nabla h} d V\right)
$$

where $\vec{q}$ is the specific discharge, $\overrightarrow{\nabla h}$ is the specific piezometric head gradient, both are defined at the fine scale, and the averaging takes place over a block of volume $V$. The definition can be interpreted as Darcy's

law at the coarse scale $\bar{q}=-\vec{K}_{V} \nabla \bar{\gamma}$, where the bar denotes volumetric average. At the same time, $\vec{q}$ and $\overrightarrow{\nabla h}$ are also related by Darcy's law but at the fine scale $\vec{q}=-\vec{k} \nabla h$. Hereon, we will use the terms "block" and "cell" to denote the basic elements at the coarse and fine scales, respectively.

Except on counted occasions, $\vec{K}_{V}$ is a three-dimensional full tensor:

$$
\vec{K}_{V}=\left(\begin{array}{lll}
K_{x x} & K_{x y} & K_{x z} \\
K_{y x} & K_{y y} & K_{y z} \\
K_{z x} & K_{z y} & K_{z z}
\end{array}\right) .
$$

The tensor must be symmetric and positive definite (Bear, 1972; Farmer, 2002; Giudici and Vassena, 2007), that is,

$$
\begin{aligned}
K_{x y}=K_{y x}, K_{x z}=K_{z x}, K_{y z} & =K_{z y} \\
K_{x x}>0, K_{y y}>0, K_{z z} & >0 \\
K_{x x} K_{y y} & >K_{x y}^{2} \\
K_{x x} K_{y y} K_{z z}+2 K_{x y} K_{x z} K_{y z}-K_{x x} K_{y z}^{2}-K_{y y} K_{x z}^{2}-K_{z z} K_{x y}^{2} & >0 .
\end{aligned}
$$


Most computer codes - particularly finite-difference codes - only admit diagonal tensors, something that requires that all blocks have the same principal directions, and that they are all aligned with the Cartesian axes. However, many natural geologic formations, e.g., cross-bedded sediments (Bierkens and Weerts, 1994), would require the use of full tensors. Even, under simple patterns of spatial heterogeneity, a full tensor description yields better results than a diagonal tensor, as will be shown later.

It is worthwhile to note that $\vec{K}_{V}$ is a function, not only of the cell values of $\vec{q}$ and $\overrightarrow{\nabla h}$ in equation (1), but also on the boundary conditions at the aquifer scale used to solve the groundwater flow equation yielding these cell values. This dependence on the boundary conditions is what makes the resulting upscaled block values non-local.

Because we cannot have the actual head values around the block boundary as each block would have under the specific aquifer geometry and boundary conditions (this would require the solution of the flow equation at the fine scale defeating the main purpose of the upscaling exercise) we have to solve many local problems extracting each block from the aquifer, applying some boundary conditions to its sides, and solving a local flow problem. We can find in the literature different choices for the boundary conditions used in the local model to compute the block values, from permeameter-type boundary condition (two sides with constant head while the others are no-flow boundaries) to prescribed heads at all block faces inducing an overall head gradient in a specific direction (e.g., see below Figure 3), to periodic boundary conditions (Pickup et al., 1994; Renard and Marsily, 1997; Wen et al., 2003). The prescribed head boundary condition on all sides of the domain is employed in the proposed procedure.

As mentioned before, the best boundary conditions that could be used to determine the block conductivities are prescribed heads equal to the ones observed around the block under the specific (global) flow conditions applied to the entire aquifer. But obtaining these head values would require the solution of the flow problem at the fine scale, beating our objective of alleviating the computing effort for solving the flow equation. As an alternative to the solution of the flow equation at the fine scale over the full domain, Gómez-Hernández (1991) proposed to use a sufficiently large "border ring" or "skin" around the block so as to reduce the impact of the artificial boundary conditions used in the upscaling procedure. By isolating the block of volume $V$ plus a skin around it and then applying prescribed heads as boundary conditions to compute the fine scale solution on the block-plus-skin domain, we let the cells that are neighboring the block control, to some extent, the flow pattern within the block; at the limit, for a skin infinitely large, the flow pattern within the block would be exactly the same as if the flow equation had been solved over the entire aquifer. Other choices for the boundary conditions would be possible but we found that prescribed heads in all sides provide excellent results in all our tests, in fact, the goodness of the approach is more sensible to the size of the skin than to the choice of boundary conditions.

Another important feature of our algorithm is that it is aimed at computing directly the interblock conductivity values used by finite-difference codes to solve the groundwater flow equation. For completeness, we offer two alternatives as shown in Figure 1: computing the block conductivity tensor, or computing directly the interface conductivity. We argue that for the purpose of reproducing the flow patterns observed at the fine scale it is better to compute directly the interblock conductivities ( $\mathrm{Li}$ et al., 2010). In the figure we can appreciate the block that is being upscaled plus a skin, a single-cell wide, surrounding it, for both cases. Since the skin is needed to determine the block values for the blocks at the edges of the aquifer, the figure also shows that there is a need to consider a skin around the coarse model, at least of the same width as the skin needed for the upscaling process.

In summary, block upscaling for a given volume within a larger domain that has been discretized into small cells is performed by isolating the cells within the block plus a sufficiently large number of skin cells surrounding it. Then, the flow equation is solved for this set of cells applying linearly varying prescribed heads along the boundaries (see Figure 3). Several boundary conditions are used inducing average piezometric head gradients at varying orientations. For each boundary condition, block specific discharges and block head gradients are computed as explained later; then, the symmetric, positive definite tensor that best reproduces the relationship between average flows and average gradients according to equation (1) is computed and used as the block conductivity. 


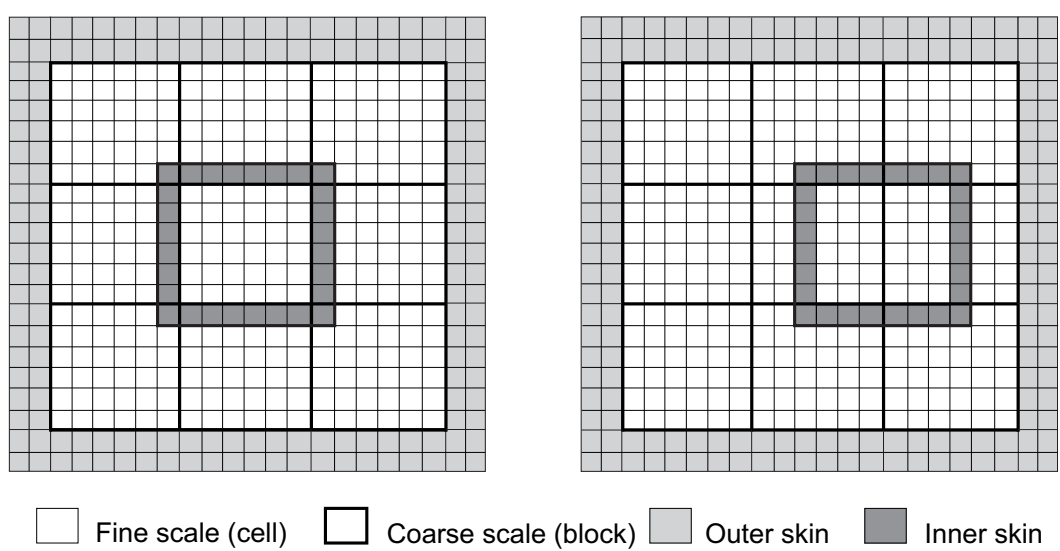

Figure 1: Schematic diagram to show the cells that will be considered to compute a block conductivity (left) or an interblock conductivity (right). In both cases, a skin of a single-cell wide around the block is used. Notice also the skin considered around the coarse aquifer model, this skin is necessary for properly computing the block values at the aquifer edges.

\subsection{Implementation details}

The algorithm can be summarized in the following steps:

1. Input parameters and fine scale field to be upscaled. As starting point we need the fine scale hydraulic conductivity realization (i.e, it could have been obtained by geostatistical simulation). At this scale, the aquifer is discretized in equal-sized parallelepiped cells. Then, we need the description of the coarse scale discretization; it can be non-uniform (see Figure 2 for a 2D example). Additional input parameters are the size of the skin to be used for upscaling given as number of cells (the same skin size will be used for all blocks), the number of flow problems that will be solved to upscale each block, for each of these problems a different set of linearly varying prescribed heads will be used, these prescribed heads are defined by a vector corresponding to the average head gradient induced in the block. A minimum of two flow problems have to be solved in 2D, and three in $3 \mathrm{D}$, in order to be able to identify all components of the block conductivity tensor, but it is advisable to use a larger number. It is important to notice that because a skin is used to upscale all blocks, even for the blocks which are at the aquifer boundaries, it is necessary that the volume occupied by the fine scale realization be larger than the volume of the coarse scale model by, at least, a number of cells equal to the skin size in all directions (Figure 1 shows a coarse scale model with three by three blocks overlaying a fine scale discretization which is two cells wider in all directions than the coarse model)

Figure 3 shows four boundary conditions used for the upscaling of a $2 \mathrm{D}$ block with indication of the average piezometric head vectors defining them.

2. Decide how to read the fine scale field depending on whether block centered or interface centered conductivity tensors are to be computed. The size of the skin will also have an influence on which values are read from the fine scale field for the computation of the block value. Notice that when computing interface centered conductivity tensors, the number of block values that have to be computed are twice the number of block centered conductivities in $2 \mathrm{D}$ and thrice the number in $3 \mathrm{D}$.

3. For each block/interblock and for each mean head gradient direction, establish the boundary conditions and solve the groundwater flow problem assuming incompressible, single phase, steady-state groundwater flow. The preconditioned conjugate-gradient scheme, amended according to Hill (1990), is used to solve the flow equation. The number of flow directions should be sufficient to allow determining the components of the conductivity tensor. In $2 \mathrm{D}$, for each set of boundary conditions we can obtain two equations relating the components of the average specific discharge through the block to the average 


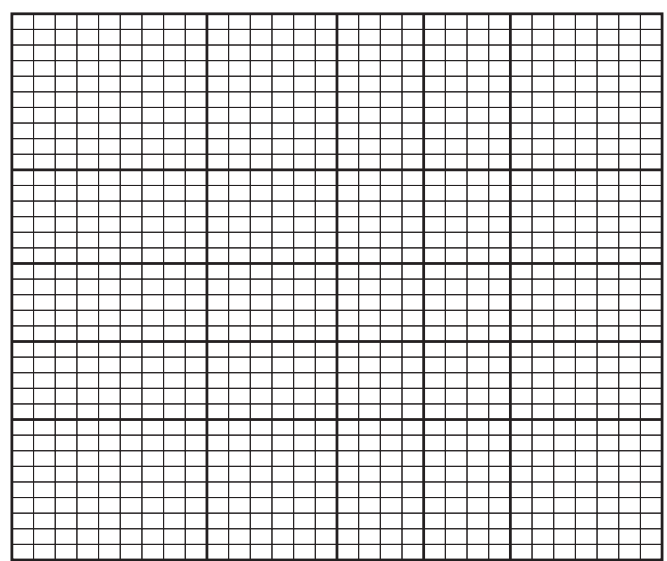

$\square \quad$ Fine scale (cell) $\square$ Coarse scale (block)

Figure 2: Schematic of non-uniform block discretization
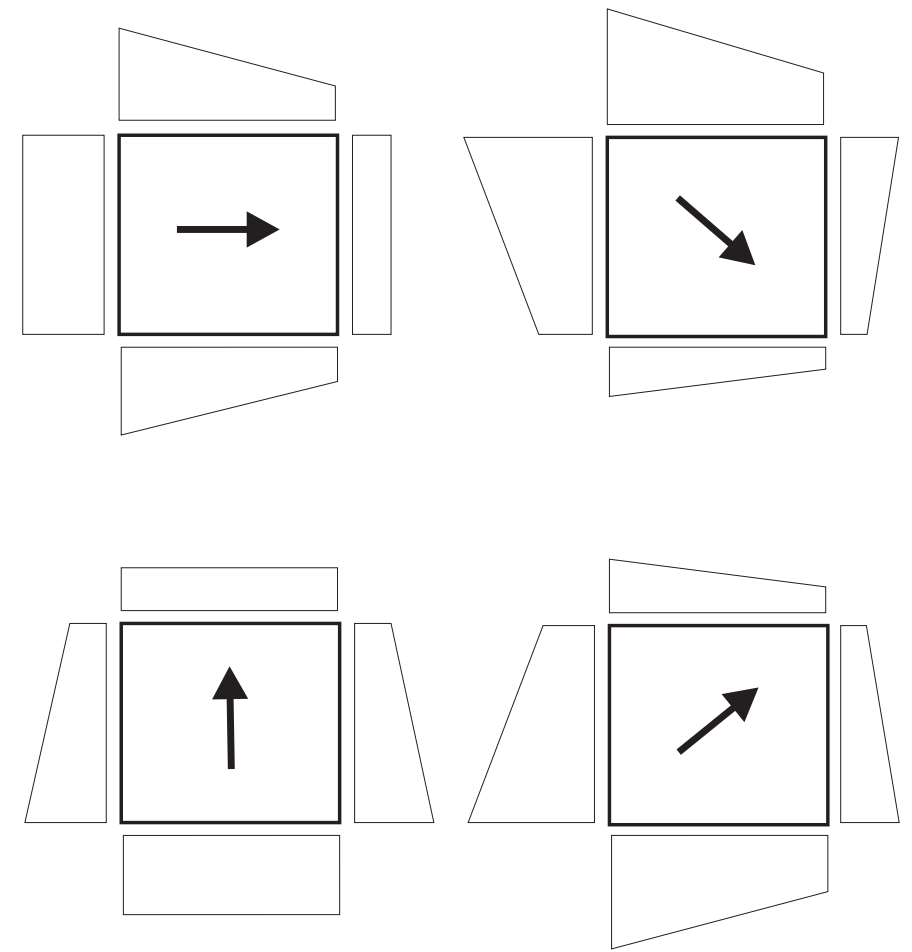

Figure 3: Four typical boundary conditions used to estimate a block conductivity in 2D 
head gradient within the block; since we have three unknown components in the conductivity tensor, there is a need to run at least two sets of boundary conditions. We recommend using, at least, four such boundary conditions.

In 3D, for each boundary condition we can establish three equations:

$$
\left(\begin{array}{l}
\bar{q}_{x} \\
\bar{q}_{y} \\
\bar{q}_{z}
\end{array}\right)=-\left(\begin{array}{lll}
K_{x x} & K_{x y} & K_{x z} \\
K_{x y} & K_{y y} & K_{y z} \\
K_{x z} & K_{y z} & K_{z z}
\end{array}\right)\left(\begin{array}{c}
\nabla \bar{h}_{x} \\
\nabla \bar{h}_{y} \\
\nabla \bar{h}_{z}
\end{array}\right),
$$

where $K_{x x}, K_{x y}, K_{x z}, K_{y y}, K_{y z}, K_{z z}$ are the unknown components of the block conductivity tensor $\vec{K}_{V}$. The remaining coefficients in this equation have different meaning depending on whether we are computing a block or an interblock tensor. For the case in which we are interested in the block tensor, $\bar{q}_{x}, \bar{q}_{y}, \bar{q}_{z}$ and $\nabla \bar{h}_{x}, \nabla \bar{h}_{y}, \nabla \bar{h}_{z}$ are the averages of specific discharge $\vec{q}$ and gradient $\nabla \vec{h}$, respectively, within the block, which can be calculated as follows:

$$
\begin{aligned}
\bar{q}_{x}=\frac{1}{n_{c}} \sum_{i=1}^{n_{c}} q_{x i}, & \nabla \bar{h}_{x} & =\frac{1}{n_{c}} \sum_{i=1}^{n_{c}} \nabla h_{x i}, \\
\bar{q}_{y}=\frac{1}{n_{c}} \sum_{i=1}^{n_{c}} q_{y i}, & \nabla \bar{h}_{y} & =\frac{1}{n_{c}} \sum_{i=1}^{n_{c}} \nabla h_{y i}, \\
\bar{q}_{z}=\frac{1}{n_{c}} \sum_{i=1}^{n_{c}} q_{z i}, & \nabla \bar{h}_{z} & =\frac{1}{n_{c}} \sum_{i=1}^{n_{c}} \nabla h_{z i},
\end{aligned}
$$

where the summations extend over all $n_{c}$ cells in the block and correspond to the fluxes or gradients between each cell and the adjacent cell in the corresponding direction.

However, when we seek the interblock conductivity (see right side of Fig. 1) since our goal will be to reproduce the fluxes across the interface, we will use a different definition of the terms in Eq. 1; more precisely, for an interblock centered on an interface orthogonal to the $x$ direction, we will take $\bar{q}_{x}$ as the specific discharge across the interface, and $\nabla \bar{h}_{x}$ as the gradient computed using the average of the piezometric heads at both sides of the interface, while for the $y$ and $z$ directions we will divide the interblock with a hypothetical interface in two equal halves, and compute the specific discharge across this interface, and the average gradient as the gradient computed using the average of the heads at both sides of the interface.

For instance, in 2D, for an interblock centered on an interface orthogonal to the $x$ direction, after solving the flow equation for a given set of boundary conditions we will compute the average fluxes and gradients as follows (see Fig. 4)

$$
\begin{aligned}
\overline{q_{x}} & =\frac{1}{n_{b x}} \sum_{i=1}^{n_{b x}} q_{x i} \\
\nabla \overline{h_{x}} & =\frac{2}{L_{x}}\left(\frac{1}{n_{h x 2}} \sum_{i 2=1}^{n_{h x 2}} h_{i 2}-\frac{1}{n_{h x 1}} \sum_{i 1=1}^{n_{h x 1}} h_{i 1}\right) \\
\overline{q_{y}} & =\frac{1}{n_{b y}} \sum_{j=1}^{n_{b y}} q_{y j} \\
\nabla \overline{h_{y}} & =\frac{2}{L_{y}}\left(\frac{1}{n_{h y 2}} \sum_{j 2=1}^{n_{h y 2}} h_{j 2}-\frac{1}{n_{h y 1}} \sum_{j 2=1}^{n_{h y 1}} h_{j 1}\right)
\end{aligned}
$$

where $n_{b x}$ is the number of cells along the interface, $q_{x i}$ are the specific discharges across the interface cells, $n_{h x 2}$ are the number of cells within the interblock to the right of the interface, and $n_{h x 1}$ are the 

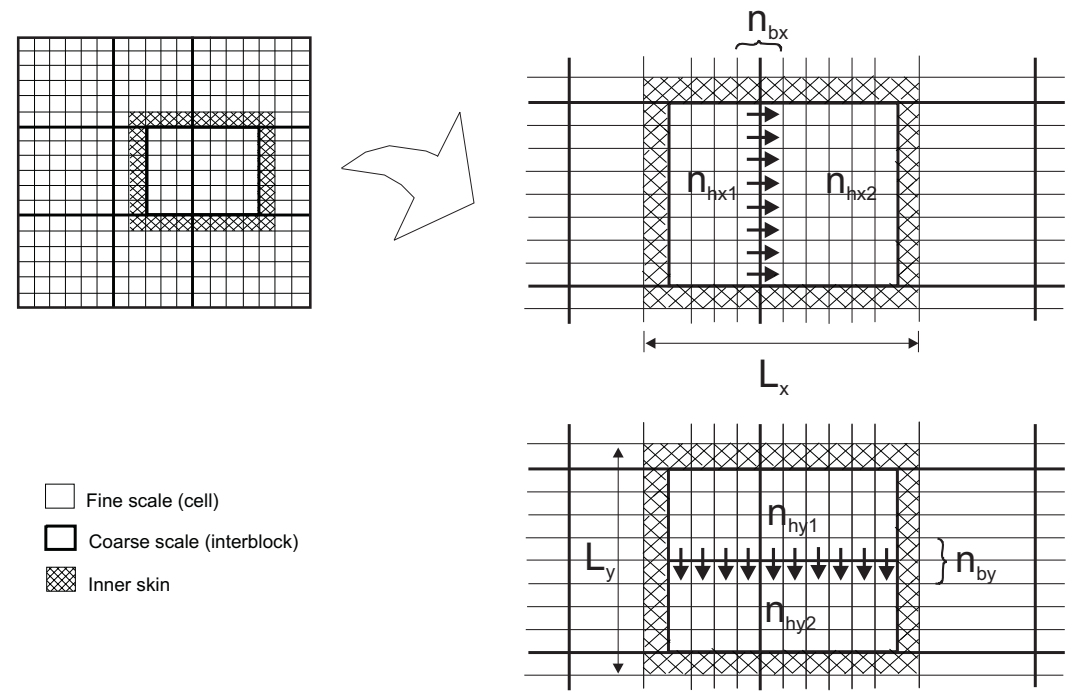

Figure 4: Computing the interblock fluxes.

number of cells to the left of the interface, $h_{i 2}$ and $h_{i 1}$ are the associated heads to the right and left of the interface respectively, $L_{x}$ is the size of the enlarged interblock in the $x$ direction; $n_{b y}$ are the number of cells along a fictitious interface running through the center of the interblock orthogonal to the $y$ direction, $n_{h y 2}$ are the number of cells below the interface (we consider that the positive $y$ direction runs downwards), $n_{h y 1}$ are the number of cells above the interface, $h_{j 2}$ and $h_{j 1}$ are the associated heads below and above the interface respectively, and $L_{y}$ is the size of the enlarged interblock in the $y$ direction.

A similar pair of equations are obtained for a block centered on an interface orthogonal to the $y$ direction.

The extension to $3 \mathrm{D}$ is straightforward.

4. Once the flow equation has been solved for the set of boundary conditions chosen (as already mentioned we recommend 4 sets in $2 \mathrm{D}$ and 8 sets in $3 \mathrm{D}$ as a minimum) we can compute all elements in vectors $\vec{q}$ and $\nabla \vec{h}$ for each of the boundary conditions. When we assemble all of these equations we arrive to an overdetermined system of linear equations from which to derive the components of $\vec{K}_{V}$.

The system of equations is shown next for the $3 \mathrm{D}$ case. Only the first six equations, corresponding to two of the boundary conditions used to solve the flow equation are explicitly shown:

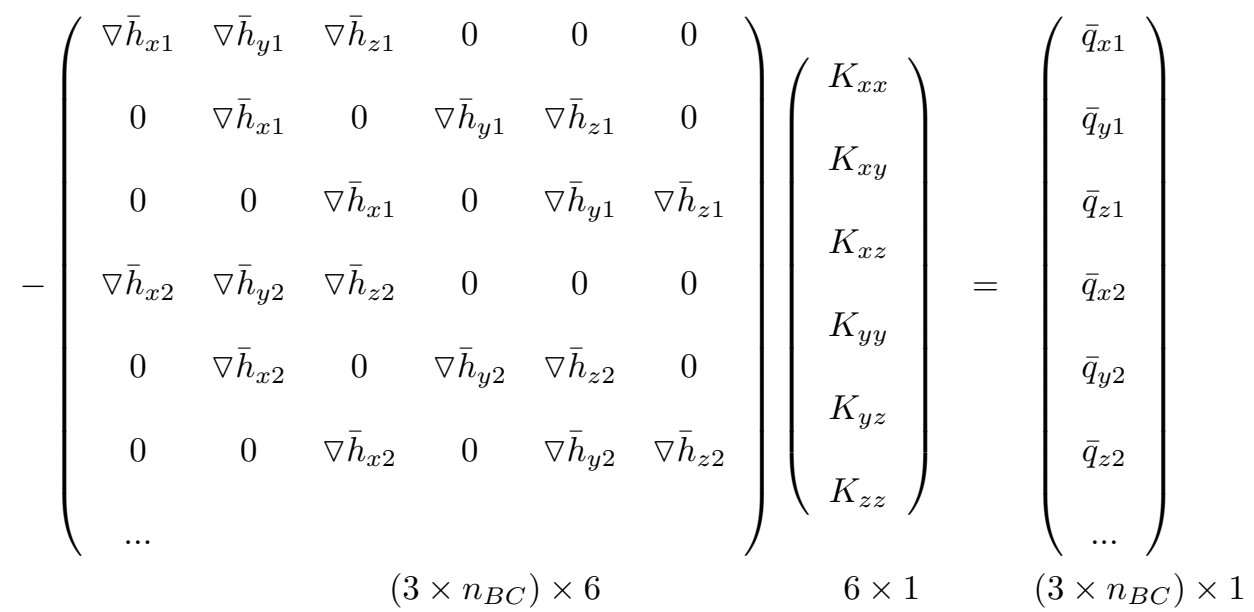


where $n_{B C}$ is the number of boundary conditions used, and the subscripts " $1,2 \ldots$ " refer to the specific boundary condition.

Standard least squares procedure is employed to solve this overdetermined system of equations (Press et al., 1988).

\section{Synthetic experiments}

\subsection{Experiment description}

To demonstrate the accuracy of the program, four synthetic experiments are carried out. In the first experiment hydraulic conductivity displays an isotropic spatial continuity and in the second and third ones hydraulic conductivity is anisotropic. In the last example the media is composed of shale and sand, characterized by a bimodal distribution mimicking a fluvial deposit. The experiments proceed as follows:

1. Generate a stochastic conductivity field by Sequential Gaussian Simulation (using GCOSIM3D (GómezHernández and Journel, 1993), or SGSIM from GSLIB (Deutsch and Journel, 1998)) over a domain discretized into 120 columns, 170 rows and 70 layers with cell size $\triangle x=\Delta y=\Delta z=1 \mathrm{~L}$ for the first three experiments (Hereon, $\mathrm{L}$ will be used as the distance measurement unit). Only the inner 100 columns by 150 rows by 50 layers will be upscaled, considering different skin sizes. The conductivities follow a $\log$-normal distribution, i.e., $\ln K \sim N(0,1)$ with an exponential variogram model. Fig. 5 shows the isotropic conductivity field used in the first experiment, in which the range is set to 20 $\mathrm{L}$ in all directions. In the second experiment the ellipsoid of anisotropy imposes ranges in the three principal directions of $80 \mathrm{~L}, 20 \mathrm{~L}$ and $5 \mathrm{~L}$. The orientation of the ellipsoid is given following the GSLIB convention (Deutsch and Journel, 1998, p. 26): align the ellipsoid with its largest axis parallel to the $Y$-axis, its medium axis parallel to the $X$-axis and its shortest axis parallel to the $Z$-axis, then rotate the ellipsoid in the $Y Z$ plane $45^{\circ}$ counter-clockwise. Fig. 6 displays the second field. The third case has the same anisotropic correlation as the second one, same zero mean, but a variance of 5 . The bimodal field is plotted in Fig.7 where the facies distribution is generated with FLUVSIM (Deutsch and Tran, 2002) and each facies is then populated by corresponding Gaussian random field (Table 1). In this experiment, we aim to test the performance of the proposed algorithm in more challenging field and further discuss the computation efficiency. For the sake of conciseness, a 2D aquifer with size 500 columns and 400 rows together with 10 skins is presented.

Table 1: Parameters of the random functions describing the two facies in the bimodal example

\begin{tabular}{cccccc}
\hline Facies & Mean & Variance & Variogram type & $\lambda_{X}^{*}(\mathrm{~L})$ & $\lambda_{Y}^{*}(\mathrm{~L})$ \\
\hline shale (background) & -2 & 1 & exponential & 15 & 15 \\
sand (channel) & 1 & 1 & exponential & 30 & 15 \\
\hline
\end{tabular}

* ranges in $\mathrm{X}$ and $\mathrm{Y}$ direction.

2. Solve the groundwater flow equation at the fine scale. The aquifers in all synthetic examples are assumed to be confined and have prescribed head boundary conditions. The prescribed heads are set so that they induce an overall diagonal head gradient. MODFLOW2000 (Harbaugh et al., 2000), which is based on an integral finite-difference method, is used to solve the flow equation at this scale. The upscaled model discretization of 10 columns by 15 rows by 5 layers is overlain on this solution (for the first three experiments) and the specific discharges crossing the coarse scale block interfaces are computed. These specific discharges will serve as the reference quantities to be matched by the upscaled model. 


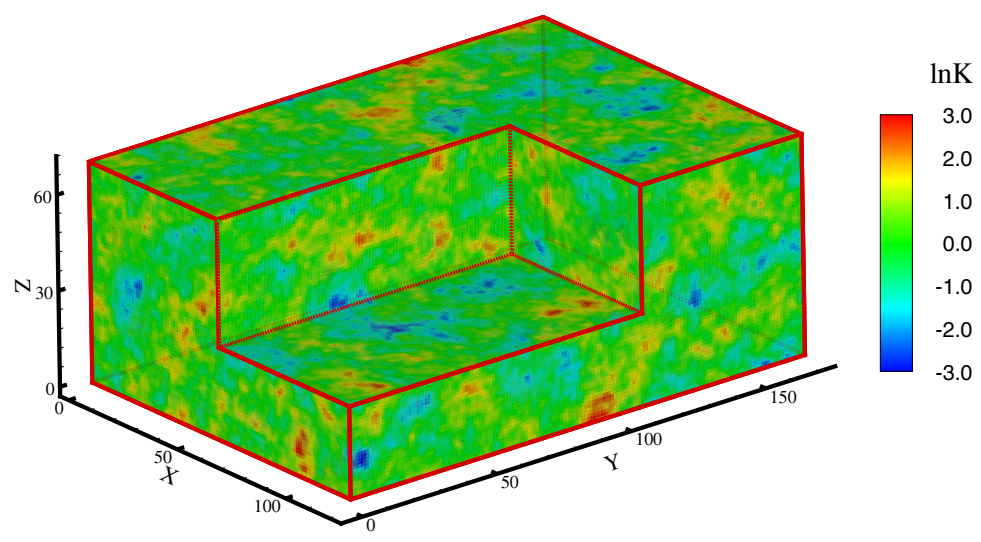

Figure 5: The reference isotropic conductivity field

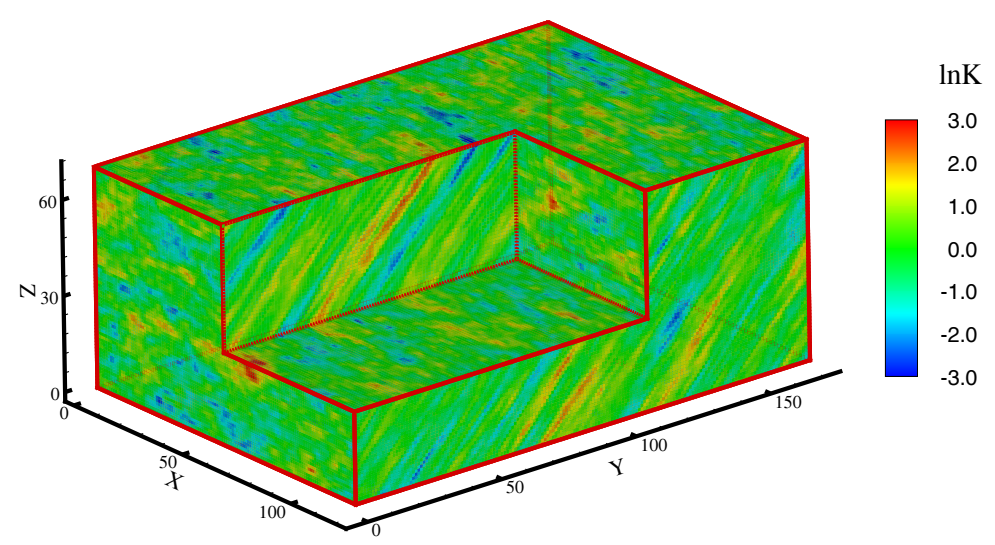

Figure 6: The reference anisotropic conductivity field

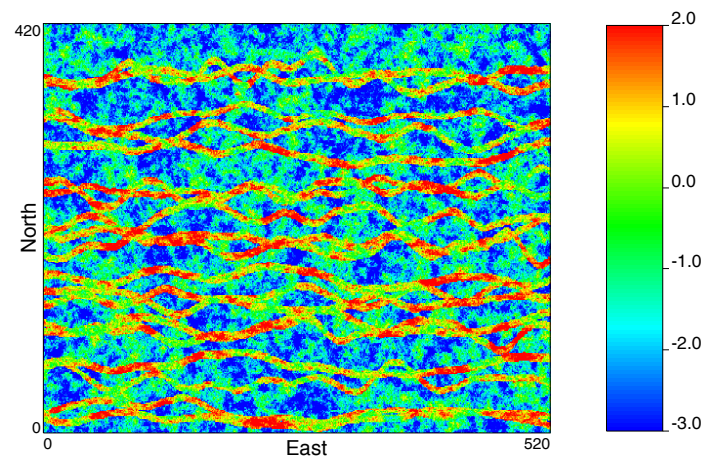

Figure 7: The reference bimodal conductivity field 
3. Perform upscaling. For the first three experiments, the inner domain of 100 columns by 150 rows by 50 layers of the fine scale conductivity field is upscaled into a coarse scale model of 10 columns by 15 rows by 5 layers, each block of size $10 \mathrm{~L} \times 10 \mathrm{~L} \times 10 \mathrm{~L}$. In the fourth case the 500 columns by 400 rows under fine scale will be coarsened to different extent, i.e., to 100 by 80,50 by 40 and 25 by 20 with corresponding block size of $5 \mathrm{~L} \times 5 \mathrm{~L}, 10 \mathrm{~L} \times 10 \mathrm{~L}$ and $20 \mathrm{~L} \times 20 \mathrm{~L}$.

The upscaled interblock conductivities will be used directly in a finite difference code (Li et al., 2010) to solve the flow equation at the coarse scale, thus avoiding any unnecessary averaging of block conductivities to determine the interblock values. In addition, we perform a sensitivity study to the skin size by comparing the results of upscaling with four different skin widths: $0,2,5$ and $10 \mathrm{~L}$ for the first three experiments.

4. Solve the groundwater flow equation at the coarse scale with the interblock conductivities computed from step 3. Determine the corresponding specific discharges at the corase scale, which will be compared with the reference values obtained in step 2. For the solution of the flow equation with generic conductivity tensors and direct input of interblock conductivities we wrote a specific numerical code. The interested read should refer to Li et al. (2010) for the details in the flow simulator.

5. Perform comparisons. We will compare the interblock fluxes at the coarse scale with those obtained in the reference simulation. For this comparison, besides graphical representations we will quantify the departure between reference and upscaled values using "Root Mean Square Error" (RMSE in short)

$$
R M S E=\sqrt{\frac{1}{N} \sum_{i=1}^{N}\left(\bar{q}_{i}^{f}-q_{i}^{c}\right)^{2}}
$$

where $\bar{q}_{i}^{f}$ and $q_{i}^{c}$ are the $i$ th interblock specific discharge at the fine and coarse scales, respectively, and $N$ is the number of interblocks computed.

\subsection{Verification of the flow reproduction}

We have chosen to show the verification of the flow rates across the block faces and the flux through the entire domain instead of the reproduction of the average piezometric head, since the head value reproduction is easier to achieve with any moderately appropriate upscaling algorithm.

\subsubsection{Isotropic conductivity field}

The comparison of the specific discharges obtained from the coarse scale and the fine scale models is presented in Fig. 8. It can be found readily from Fig. 8 that the specific discharge is well reproduced in the upscaled model even when it is computed using a block value that has been computed with no skin or with a small skin. At the same time, with the increase of skin size, the specific discharges at the coarse scale approximate better those of the fine scale. The RMSEs of specific discharge resulting from the simulations for different skins are listed in Table 2. RMSEs of $\bar{q}_{x}, \bar{q}_{y}, \bar{q}_{z}$ decrease as the skin grows, confirming the fact that computation of block conductivity should be non-local, i.e., it should account for the conductivities surrounding the block being upscaled. It is worth noting that the RMSEs for a skin of size $10 \mathrm{~L}$ did not exhibit any notable improvement compared with that for a skin of 5 L. From a computational point of view, the computer time needed for the upscaling grows exponentially with the size of the skin, thus it is important to balance the upscaling quality and the computational cost.

In this example, a skin of $5 \mathrm{~L}$ is sufficient to produce a good upscaling. We should notice that the size of the local model that is analyzed to perform the upscaling for this skin size is $20 \mathrm{~L} \times 20 \mathrm{~L} \times 20 \mathrm{~L}$, which coincides with the correlation length of the fine scale field. As it is well known, whenever the blocks are larger than the correlation scales, the flow pattern is mostly determined by the conductivities within the blocks and not so much by the conductivities outside and boundary conditions (Gómez-Hernández, 1991). From our experience we have found that with a skin size equal to half the block size is generally enough to get good flow upscaling results. 

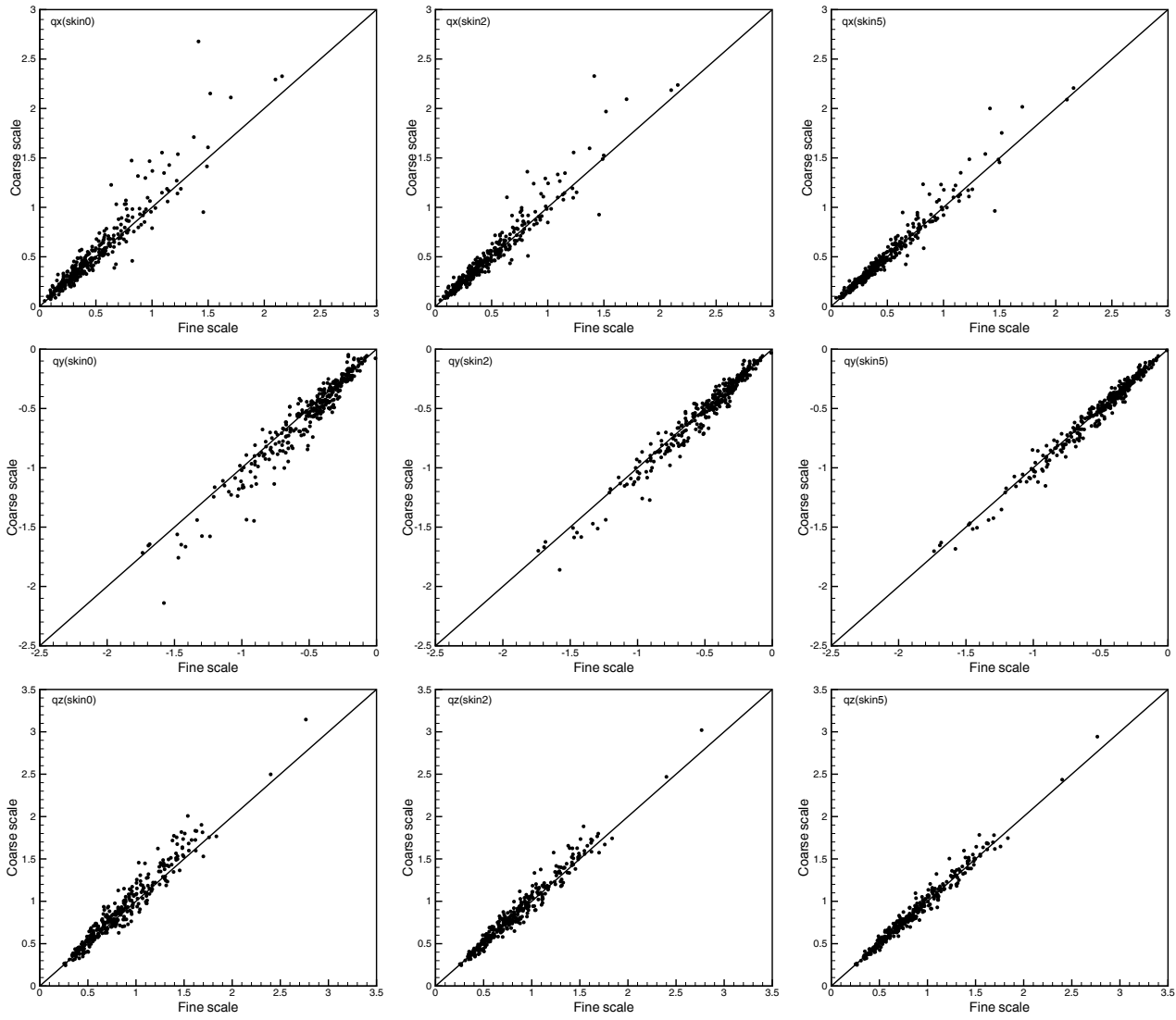

Figure 8: Specific discharges obtained with upscaled blocks using different skin sizes for the isotropic field. Comparison with the results from fine scale simulations. The graphs show, from top to bottom $\bar{q}_{x}, \bar{q}_{y}$ and $\bar{q}_{z}$, and from left to right skin sizes of 0,2 and 5 fine scale cells.

Table 2: Root Mean Square Error (RMSE) of the coarse scale specific discharge in the isotropic case

\begin{tabular}{ccccc}
\hline skin size & 0 & $2 \mathrm{~L}$ & $5 \mathrm{~L}$ & $10 \mathrm{~L}$ \\
\hline $\bar{q}_{x}$ & 0.145 & 0.111 & 0.082 & 0.074 \\
$\bar{q}_{y}$ & 0.112 & 0.075 & 0.052 & 0.046 \\
$\bar{q}_{z}$ & 0.119 & 0.084 & 0.062 & 0.056 \\
\hline
\end{tabular}




\subsubsection{Anisotropic conductivity field}

Fig. 9 is used to illustrate the performance of the upscaling computer code in an anisotropic conductivity field. Compared with the isotropic example, the upscaled conductivity in the anisotropic field is more sensitive to the size of the skin and the flux reproduction is noticeably improved when a larger skin is employed. RMSEs for $\bar{q}_{x}, \bar{q}_{y}, \bar{q}_{z}$ are presented in Table 3. We found that the RMSEs in the anisotropic field are comparable with those in isotropic field (Table 2), indicating that the proposed upscaling technique works well in both isotropic and anisotropic cases.

As in the isotropic case, a skin size of $5 \mathrm{~L}$ (half the block size) is enough to obtain good upscaling results. In this particular case, the local model size $(20 \mathrm{~L} \times 20 \mathrm{~L} \times 20 \mathrm{~L})$ is smaller than the correlation length in the direction of maximum continuity and larger than the correlation length in the minimum continuity directions. (Recall the correlation length in the three principal directions are $80 \mathrm{~L}, 20 \mathrm{~L}$ and $5 \mathrm{~L}$, and they are not all aligned with the Cartesian axes.)

The previous two synthetic experiments are characterized by a moderate variance, i.e., $\sigma_{\ln K}^{2}=1$. We have also tested the code with an anisotropic field of variance 5 (similar to the one found, for instance, at the MADE site (Rehfeldt et al., 1992)). The specific discharge reproduction and RMSEs are shown in Fig. 10 and Table 3, respectively. The larger the skin, the better the reproduction. In this particular case, going up to a skin of $10 \mathrm{~L}$ improves the reproduction with respect to the results using a skin of $5 \mathrm{~L}$.
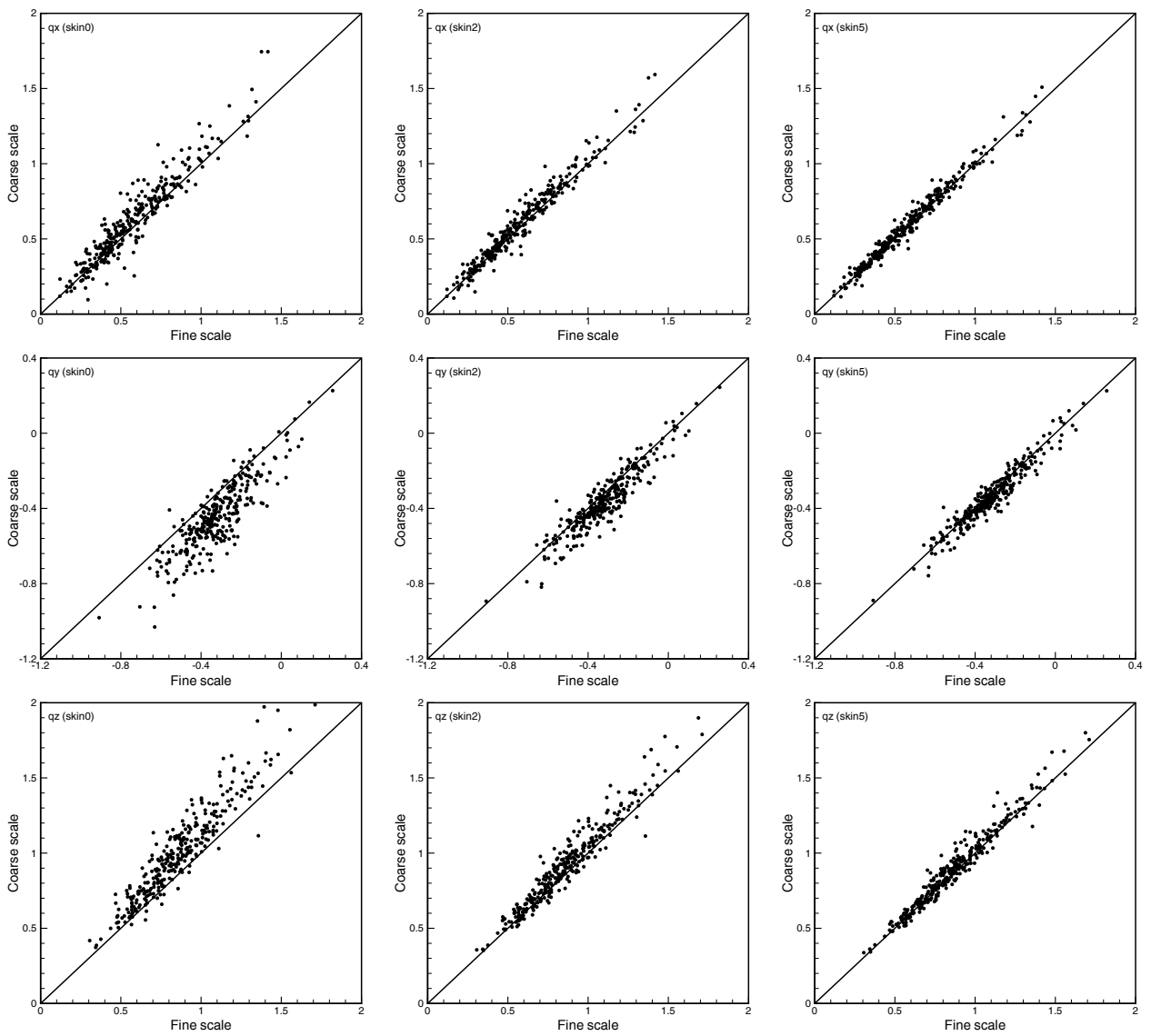

Figure 9: Specific discharges obtained with upscaled blocks using different skin sizes for the anisotropic field. Comparison with the results from fine scale simulations. The graphs show, from top to bottom $\bar{q}_{x}, \bar{q}_{y}$ and $\bar{q}_{z}$, and from left to right skin sizes of 0,2 and 5 fine scale cells. 
Table 3: Root Mean Square Error (RMSE) of the coarse scale specific discharge in the anisotropic for different skins and different variances

\begin{tabular}{cccccc}
\hline & skin size & 0 & $2 \mathrm{~L}$ & $5 \mathrm{~L}$ & $10 \mathrm{~L}$ \\
\hline \multirow{3}{*}{$\sigma_{\operatorname{lnK}}^{2}=1$} & $\bar{q}_{x}$ & 0.100 & 0.062 & 0.041 & 0.036 \\
& $\bar{q}_{y}$ & 0.154 & 0.073 & 0.046 & 0.041 \\
& $\bar{q}_{z}$ & 0.180 & 0.090 & 0.060 & 0.052 \\
\hline \multirow{3}{*}{$\sigma_{\ln K}^{2}=5$} & $\bar{q}_{x}$ & 0.944 & 0.495 & 0.325 & 0.262 \\
& $\bar{q}_{y}$ & 0.693 & 0.310 & 0.236 & 0.229 \\
& $\bar{q}_{z}$ & 0.947 & 0.370 & 0.237 & 0.225 \\
\hline
\end{tabular}

\subsubsection{Bimodal conductivity field}

The main interest of the bimodal conductivity field mimicking a fluvial deposit is that the principal directions of the upscaled block conductivities will clearly change from one block to another according to the local orientation of the meander. We have also used this example to see how results evolve as coarser and coarser upscaling is performed.

In this experiment the fine conductivity field with 500 by 400 cells is upscaled to 100 by 80,50 by 40 and 25 by 20, with increasing block sizes at the coarse scale from $5 \times 5$ cells, to $10 \times 10$ cells and $20 \times$ 20 cells. The boundary conditions induce an overall gradient from the upper left corner to the lower right one, so that there is significant flux across all block interfaces. Fig. 11 shows the comparison of the specific discharges obtained at different coarse scales compared to the fine scale. The upscaled hydraulic conductivity tensors are calculated with a skin size of 5 cells. The specific discharges across the block interfaces are well reproduced in all three coarsening levels as shown in Fig. 11.

Besides checking the reproduction of specific discharge, we furthermore compared the total flux across the entire domain. The results are listed in Table 4 . The reproduction by the upscaled models of the total flux crossing a vertical section $\left(q_{x}\right)$ or a horizontal section $\left(q_{y}\right)$ through the aquifer center is very good for $q_{x}$ and shows some overestimation for $q_{y}$, which increases as the coarse blocks get larger. This behavior is clearly related to the main orientation of the channels in the aquifer, since similar tests without the channels and similar degree of heterogeneity result in equally good reproduction of the total fluxes both for the horizontal and the vertical directions.

To demonstrate the computational cost of the proposed algorithm, we recorded the runtime of upscaling from 500 by 400 to different coarse scales with varying skin sizes (Table 5) in a personal computer with a processor running at $2.21 \mathrm{GHz}$ and $1.00 \mathrm{~GB}$ of RAM. As we have already mentioned in the previous experiment, the upscaling with large skins requires longer computation time than with small ones, since for each upscaled block the local flow problem is larger. While larger block sizes, imply less number of total blocks to upscale, which compensate the fact that the local flow problems will be larger, and therefore demand less time. Thus, computational efficiency demands a careful choice between the block size and the skin. 

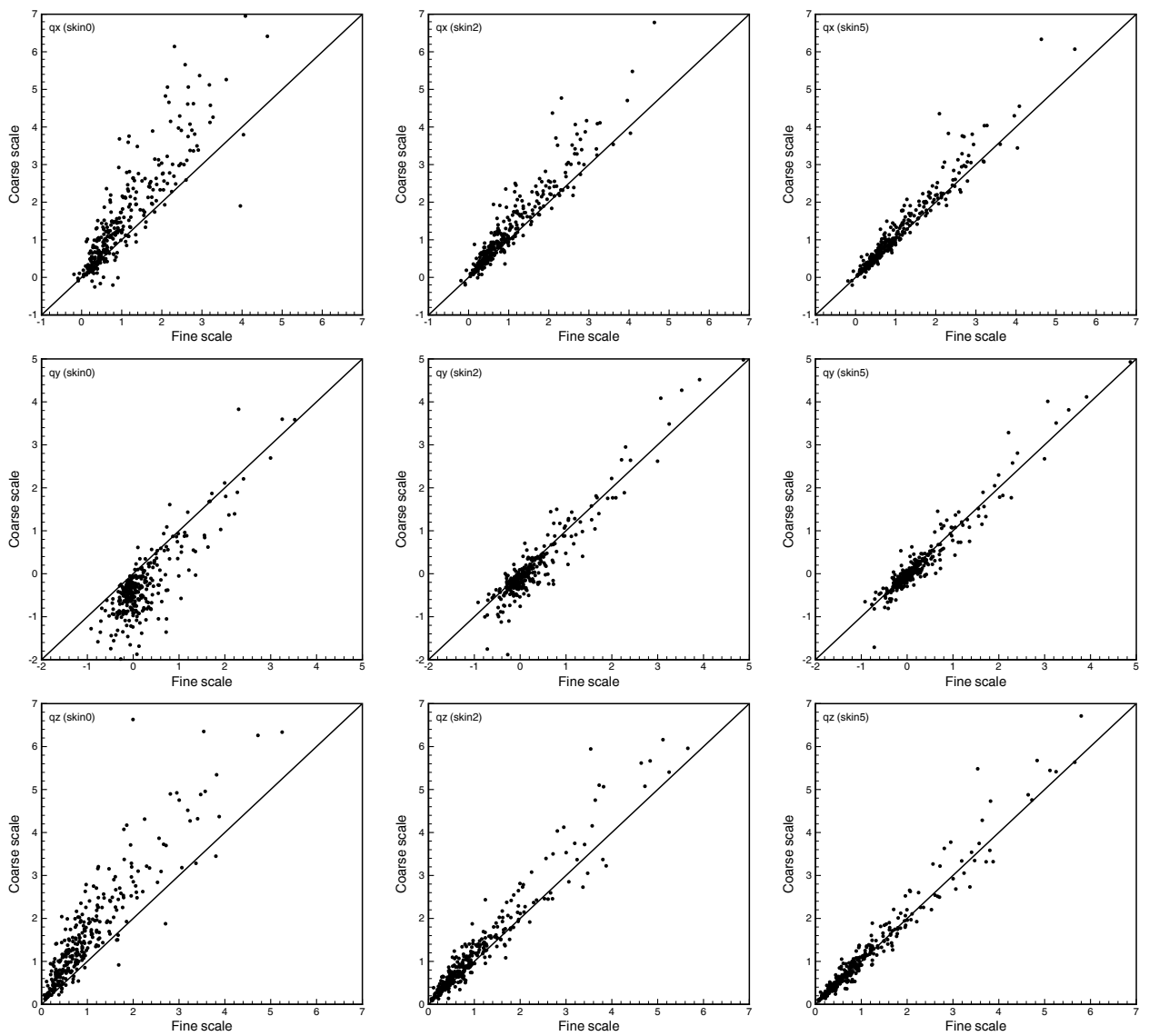

Figure 10: Reproduction of specific discharge, from top to bottom we show $\bar{q}_{x}, \bar{q}_{y}$ and $\bar{q}_{z}$, and from left to right we show skin size 0,2 and 5 . The heterogeneity of the field is large, $\sigma_{\ln K}^{2}=5$. 

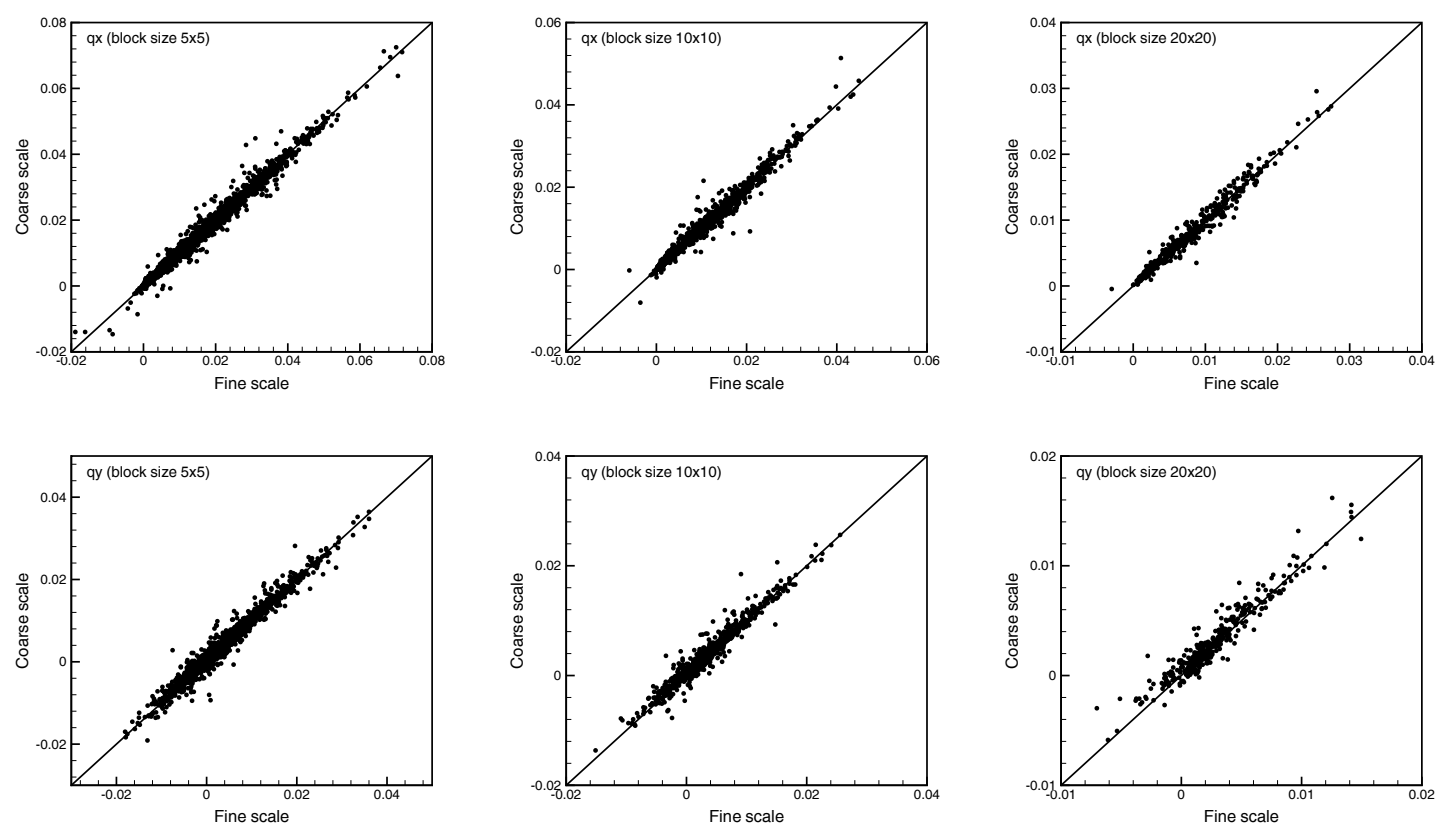

Figure 11: Specific discharges $\bar{q}_{x}$ in the first row and $\bar{q}_{y}$ in the second row obtained with upscaled blocks with skin width 5 L for the channelized field. Comparison with the results from fine scale simulations. From left to right we show comparison under different coarsening level, i.e., block size $5 \mathrm{~L} \times 5 \mathrm{~L}, 10 \mathrm{~L} \times 10 \mathrm{~L}$ and $20 \mathrm{~L} \times 20 \mathrm{~L}$.

Table 4: Total flux across the entire width of the domain

\begin{tabular}{lcccc}
\hline & \multicolumn{2}{c}{ Total flux $\left(L^{3} T^{-1}\right)$} & \multicolumn{2}{c}{ relative bias (\%) } \\
\cline { 2 - 5 } & $\bar{q}_{x}$ & $\bar{q}_{y}$ & $\bar{q}_{x}$ & $\bar{q}_{y}$ \\
\hline Fine scale $500 \times 400$ & 28.72 & 11.54 & & \\
Coarse scale $100 \times 80$ & 28.61 & 11.88 & 0.37 & 2.8 \\
Coarse scale $50 \times 40$ & 28.87 & 12.17 & 0.51 & 5.4 \\
Coarse scale $25 \times 20$ & 28.95 & 12.33 & 0.81 & 6.8 \\
\hline
\end{tabular}

Table 5: Computating time (second) consumed by different coarsening level with varying skin size

\begin{tabular}{lccc}
\hline skin size & $2 \mathrm{~L}$ & $5 \mathrm{~L}$ & $10 \mathrm{~L}$ \\
\hline Coarse scale $100 \times 80$ & 30.062 & 112.656 & 401.078 \\
Coarse scale $50 \times 40$ & 20.156 & 51.672 & 158.266 \\
Coarse scale $25 \times 20$ & 20.703 & 38.125 & 87.657 \\
\hline
\end{tabular}




\section{Conclusions}

A three-dimensional hydraulic conductivity full tensor upscaling algorithm and code was proposed in this paper. This work is an extension of the work by Gómez-Hernández (1991) in two dimensions. One of the critical features of this program resides in the capability of obtaining not only block conductivities at the center of blocks but also interblock conductivity at the interfaces. By computing directly interblock conductivities, we avoid the averaging of neighboring block values to come up with the interface conductivity needed by finite-difference codes. The verification of the algorithm requires the development of a groundwater flow solver that can handle interblock tensor conductivities. This code is also available and is described in an accompanying paper ( $\mathrm{Li}$ et al., 2010). The importance of accounting for the cells surrounding the block being upscaled has been demonstrated, proving the non-local nature of upscaled conductivities. We found that a skin size which is half the block size is enough to produce good results in the two examples with moderate variance discussed in the paper and it should be increased if the approach is applied to highly heterogeneous conductivity field.

The method consistently gives good results for all coarsening levels analyzed. This good reproduction of the simulated results has to be understood that is for the quantities computed at the coarse scale. After upscaling, we lose the small variability within the upscaled blocks, and that cannot be reproduced. That is, as the upscaling gets coarser the method performance does not have to deteriorate necessarily, however, the results we get are representative only of the coarse blocks we are modeling.

Four synthetic hydraulic conductivity fields were generated with isotropic, anisotropic and bimodal spatial correlations, and different variances. In all cases, the proposed algorithm produced remarkable results, considering that the upscaling process amounted to a loss of resolution of three orders of magnitude, i.e., each group of 10 by 10 by 10 cells was replaced by a singe homogeneous block for the first three examples. In the last experiment, we discussed the effect of different coarsening level on flow reproduction and measured the computation cost of upscaling.

The proposed upscaling algorithm is based on the numerical solution of a local groundwater flow model over an area that includes the block being upscaled plus a surrounding skin. There are as many local groundwater flow solutions as interfaces in the coarse model. This way of upscaling implies a computational burden much larger than empirical results in which each block is replaced by some type of average of the cells within the block, but, undoubtedly produces more accurate results. Care has to be taken in using a skin size large enough to capture the flow response of the block, but as small as possible to reduce the computational cost.

Acknowledgements. The authors gratefully acknowledge the financial support by ENRESA (project 0078000067) and the European Commission (projects FUNMIG and PAMINA). The first author also acknowledges the financial aid through a fellowship from the China Scholarship Council (CSC).

\section{References}

Attinger, S., Eberhard, J., Neuss, N., Oct. 2002. Filtering procedures for flow in heterogeneous porous media: numerical results. Computing and Visualization in Science. 5 (2), 67-72.

Bear, J., 1972. Dynamics of fluids in porous media. American Elsevier Pub. Co., New York. 764pp.

Bierkens, M. F. P., Weerts, H. J. T., 1994. Block hydraulic conductivity of cross-bedded fluvial sediments. Water Resources Research. 30 (10), 2665-2678.

Dagan, G., 1986. Statistical theory of groundwater flow and transport: Pore to laboratory, laboratory to formation, and formation to regional scale. Water Resources Research. 22 (9), 120S-134S.

Dagan, G., 1989. Flow and Transport in Porous Formations. Springer Verlag GmbH \& Co KG. 470pp. 
Deutsch, C. V., Journel, A. G., 1998. GSLIB, Geostatistical Software Library and User's Guide, 2nd Edition. Oxford University Press, New York. 384pp.

Deutsch, C. V., Tran, T. T., 2002. Fluvsim: a program for object-based stochastic modeling of fluvial depositional systems. Computers and Geosciences. 28 (4), 525-535.

Farmer, C. L., 2002. Upscaling: a review. International Journal for Numerical Methods in Fluids. 40 (1-2), $63-78$.

Freeze, R. A., Cherry, J. A., 1979. Groundwater. Prentice-Hall. 604pp.

Gautier, Y., Noetinger, B., 1997. Preferential flow-paths detection for heterogeneous reservoirs using a new renormalization technique. Transport in Porous Media. 26 (1), 1-23.

Gelhar, L. W., Axness, C. L., Feb. 1983. Three dimensional stochastic analysis of macrodispersion in aquifers. Water Resources Research. 19 (1), 161-180.

Giudici, M., Vassena, C., 2007. About the symmetry of the upscaled equivalent transmissivity tensor. Mathematical Geology. 39 (4), 399-408.

Gómez-Hernández, J. J., 1991. A stochastic approach to the simulation of block conductivity values conditioned upon data measured at a smaller scale. Ph.D. thesis, Stanford University. 351pp.

Gómez-Hernández, J. J., Journel, A. G., 1993. Joint sequential simulation of multigaussian fields. In: Soares, A. (Ed.), Geostatistics Tróia '92. Kluwer Academic Publishers, pp. 85-93.

Gómez-Hernández, J. J., Wen, X., 1994. Probabilistic assessment of travel times in groundwater modeling. Stochastic Hydrology and Hydraulics. 8 (1), 19-56.

Harbaugh, A. W., Banta, E. R., Hill, M. C., McDonald, M. G., 2000. MODFLOW-2000, the U.S. Geological Survey modular ground-water model. English. U.S. Geological Survey, Branch of Information Services, Reston, VA, Denver, CO.

Hill, M. C., 1990. Preconditioned conjugate-gradient 2 (PCG2), a computer program for solving ground-water flow equations. US Geological Survey Water Resources Investigations Report. 90-4048, 43pp.

Indelman, P., Dagan, G., 1993. Upscaling of permeability of anisotropic heterogeneous formations: 1.the general framework. Water Resources Research. 29 (4), 917-923.

Lake, L. W., 1988. The origins of anisotropy. Journal of Petroleum Technology. 40 (4), 395-396.

Landau, L. D., Lifshitz, E. M., 1960. Electrodynamics of continuous media. Elsevier, New York.

Li, L. P., Zhou, H. Y., Gómez-Hernández, J. J., 2010. Steady-state groundwater flow modeling with full tensor conductivities using finite differences. Computers and Geosciences. Under review.

Matheron, G. F., 1967. éléments pour une Théorie des Milieux Poreux. Mason Et Cie.

Neuman, S. P., Orr, S., 1993. Prediction of steady state flow in nonuniform geologic media by conditional moments:exact nonlocal formalism,effective conductivities, and weak approximation. Water Resources Research. 29 (2), 341-364.

Notinger, B., Artus, V., Zargar, G., Mar. 2005. The future of stochastic and upscaling methods in hydrogeology. Hydrogeology Journal. 13 (1), 184-201.

Pickup, G., Ringrose, P., Jensen, J., Sorbie, K., Feb. 1994. Permeability tensors for sedimentary structures. Mathematical Geology. 26 (2), 227-250. 
Press, W. H., Flannery, B. P., Teukolsky, S. A., Vetterling, W. T., 1988. Numerical recipes in C. Cambridge University Press, Cambridge. 735pp.

Rehfeldt, K. R., Boggs, J. M., Gelhar, L. W., 1992. Field study of dispersion in a heterogeneous aquifer 3. geostatistical analysis of hydraulic conductivity. Water Resources Research. 28 (12), 3309-3324.

Renard, P., Marsily, G. D., 1997. Calculating equivalent permeability: A review. Advances in Water Resources. 20 (5-6), 253-278.

Rubin, Y., Gómez-Hernández, J. J., 1990. A stochastic approach to the problem of upscaling of conductivity in disordered media, theory and unconditional numerical simulations. Water Resources Research. 26 (4), $691-701$.

Sanchez-Vila, X., Guadagnini, A., Carrera, J., 2006. Representative hydraulic conductivities in saturated groundwater flow. Reviews of Geophysics. 44, RG3002.

Vermeulen, P. T. M., te Stroet, C. B. M., Heemink, A. W., 2006. Limitations to upscaling of groundwater flow models dominated by surface water interaction. Water Resources Research. 42 (10), W10406.

Wen, X., Durlofsky, L., Edwards, M., Jul. 2003. Use of border regions for improved permeability upscaling. Mathematical Geology. 35 (5), 521-547.

Wen, X., Gómez-Hernández, J. J., 1996. Upscaling hydraulic conductivities: An overview. Journal of Hydrology. 183 (1-2), ix-xxxii. 